\title{
Root-Mean-Square Gains of Switched Linear Systems*
}

\author{
Technical Report
}

\author{
João P. Hespanha \\ hespanha@ece.ucsb.edu \\ Dept. Electrical and Computer Engineering \\ University of California \\ Santa Barbara, CA 93106-9560 \\ phone: (805) 893-7042, fax: (805) 893-3262
}

September 24, 2002

\begin{abstract}
In this paper we compute the root-mean-square (RMS) gain of a switched linear system when the interval between consecutive switchings is large. The algorithm proposed is based on the fact that a given constant $\gamma$ provides an upper bound on the RMS gain whenever there is a separation between the stabilizing and the antistabilizing solutions to a set of $\gamma$-dependent algebraic Riccati equations. The motivation for this problem is the application of robust stability tools to the analysis of hybrid systems.
\end{abstract}

\section{Introduction}

A switched linear system is defined by a linear differential equation for which the matrices that appear in the right-hand-side are piecewise constant. The times at which these matrices are discontinuous are called "switching times." Formally, to define a switched linear system one considers a family of $n$-dimensional, $m$-input, $k$-output minimal realizations

$$
\left\{\left(A_{p}, B_{p}, C_{p}, D_{p}\right): p \in \mathcal{P}\right\}
$$

parameterized by an index set $\mathcal{P}$, together with a family of piecewise constant signals

$$
\mathcal{S}:=\{\sigma:[0, \infty) \rightarrow \mathcal{P}\}
$$

For a given signal $\sigma \in \mathcal{S}$, one can then define the following linear time-varying system

$$
\dot{x}=A_{\sigma(t)} x+B_{\sigma(t)} u, \quad y=C_{\sigma(t)} x+D_{\sigma(t)} u .
$$

${ }^{*}$ This material is based upon work supported by the National Science Foundation under the Grant No. ECS0093762 . 
The signal $\sigma$ is called a switching signal and the times at which it is discontinuous are called switching times. Between switching times the dynamics of (2) are time-invariant. Typically, switching signals are generated by a supervisory logic and different values of $\sigma$ correspond to distinct modes of operation [1]. In this paper we consider switched systems for which all the matrices $A_{p}, p \in \mathcal{P}$ are Hurwitz. For simplicity of notation, in the sequel we drop the time-dependence in $\sigma(t)$ when the switching signal is used as a subscript to the matrices in (1).

For different switching signals $\sigma$, the system (2) has different properties. However, it is useful to study properties of this system that hold for every $\sigma$ in a particular set of switching signals $\mathcal{S}$. Stability is a property that has been extensively investigated for several sets of switching signals: We say that (2) is uniformly exponentially stable over $\mathcal{S}$ when the state transition matrix $\Phi_{\sigma}(t, \tau)$ of the switched system $\dot{z}=A_{\sigma} z$ can be bounded by ${ }^{1}$

$$
\left\|\Phi_{\sigma}(t, \tau)\right\| \leq c e^{-\lambda(t-\tau)}, \quad \forall t, \tau \geq 0, \quad \forall \sigma \in \mathcal{S},
$$

where the constants $c, \lambda>0$ are chosen independently of $\sigma$ in $\mathcal{S}$. In $[2,3,4,5,6,7,8,9,10]$ conditions are presented that guarantee stability of (2) for every set of switching signals $\mathcal{S}$. It is also well known (cf., e.g., [11]) that (2) is uniformly exponentially stable as long as $\mathcal{S}$ only contains signals with consecutive switching times separated by a sufficiently long interval. In particular, defining $\mathcal{S}\left[\tau_{D}\right], \tau_{D}>0$ to be the set of switching signals with interval between consecutive discontinuities no smaller than $\tau_{D}$, there exists a $\tau_{D}^{*}>0$ so that (2) is uniformly exponentially stable over any $\mathcal{S}\left[\tau_{D}\right]$, $\tau_{D} \geq \tau_{D}^{*}$. The minimum interval $\tau_{D}$ between consecutive switching times is called the dwell-time. In [12] it is shown that uniform exponential stability is preserved when the set of switching signals is enlarged to contain signals that occasionally have dwell times smaller than $\tau_{D}$, provided that this does not happen on-the-average (slow-switching on-the-average). The reader is referred to [13, 1$]$ for a discussion of these and related results.

Surprisingly, there has been relatively little work on the study of input-output properties that hold over sets of switching signals. A notable exception is the study of passivity-like conditions for switched systems in [14]. In this paper we study the root-mean-square (RMS) gain of a switched system $[15,12,16]$. RMS gains are typically needed to evaluate the performance of closed-loop systems with hybrid controllers or processes. This analysis also finds application in the use of robust control techniques in the stability analysis of hybrid systems.

Given a set of switching signals $\mathcal{S}$, we define the $R M S$ gain of (2) over $\mathcal{S}$ to be the smallest scalar $\mathfrak{g}_{\mathcal{S}} \geq 0$ for which

$$
\mathfrak{g}_{\mathcal{S}}:=\inf \left\{\gamma \geq 0:\|y\|_{2} \leq \gamma\|u\|_{2}, \forall u \in \mathcal{L}_{2}, \sigma \in \mathcal{S}\right\},
$$

where $y$ is computed along solutions to $(2)$ with $x(0)=0$. The RMS gain $\mathfrak{g}_{\mathcal{S}}$ can be viewed as a "worst-case" energy amplification gain for the switched system, over all possible input and switching signals.

When (2) is uniformly exponentially stable over $\mathcal{S}$, the RMS gain $\mathfrak{g}_{\mathcal{S}}$ is known to be finite. Since both switching with large dwell-time and slow-switching on-the-average result in exponential stability, either of these forms of slow switching result in a finite RMS gain $\mathfrak{g}_{\mathcal{S}}$. In $[15,12,16]$

\footnotetext{
${ }^{1}$ Given a vector $a$ and a matrix $A$ we denote by $\|a\|$ and $\|A\|$ the Euclidean norm of $a$ and the largest singular value of $A$, respectively. Given a vector or matrix-valued measurable signal $z$ defined on $[0, \infty)$, we denote by $\|z\|_{2}$ the $\mathcal{L}_{2}$-norm $\left(\int_{0}^{\infty}\|z(t)\|^{2} d t\right)^{\frac{1}{2}}$ of $z$. The set of signals $z$ for which $\|z\|_{2}$ is finite is denoted by $\mathcal{L}_{2}$.
} 
one can find upper bounds on RMS gains over appropriately defined sets of switching signals that satisfy the slow-switching properties mentioned above. However, these bounds are typically very conservative and prompted us to compute the precise value of the RMS gain under slow-switching.

The RMS gain over the set of switching signals $\mathcal{S}\left[\tau_{D}\right]$ with dwell-time $\tau_{D}>0$ is a monotone decreasing function of $\tau_{D}$. This is simply because, given two dwell-times $\tau_{D_{1}} \leq \tau_{D_{2}}$, we have that $\mathcal{S}\left[\tau_{D_{1}}\right] \supset \mathcal{S}\left[\tau_{D_{2}}\right]$. One can then ask: What is the smallest $R M S$ gain that can be obtained for (2) by increasing the dwell-time? To answer this question we define the slow-switching RMS gain, by

$$
\mathfrak{g}_{\text {slow }}:=\inf _{\tau_{D}>0} \mathfrak{g}_{\mathcal{S}\left[\tau_{D}\right]}
$$

Since every $\mathcal{S}\left[\tau_{D}\right], \tau_{D}>0$ contains the constant switching signals $\sigma(t)=p, t \geq 0, p \in \mathcal{P}$, $\mathfrak{g}_{\text {slow }}$ is necessarily larger or equal to the RMS gains of all the "unswitched systems," i.e.,

$$
\mathfrak{g}_{\text {slow }} \geq \mathfrak{g}_{\text {static }}:=\sup _{p \in \mathcal{P}}\left\|C_{p}\left(s I-A_{p}\right)^{-1} B_{p}+D_{p}\right\|_{\infty}
$$

where $\|T\|_{\infty}$ denotes the $\mathcal{H}_{\infty}$-norm of a transfer matrix $T$, i.e., $\|T\|_{\infty}:=\sup _{\Re[s] \geq 0}\|T(s)\|$. We recall that $\|T\|_{\infty}$ is numerically equal to the RMS gain of any linear time-invariant system with transfer matrix $T$. Until recently, it was believed that the inequality in (4) was actually an equality. This would mean that, by switching sufficiently slow, one would recover the RMS gains of the "unswitched systems," which are realization independent. It turns out that this is not true and the above inequality is often strict. The following simple example illustrates this and also provides some intuition on the difficulties introduced by switching: Consider a SISO switched system

$$
\dot{x}=A_{\sigma} x+b_{\sigma} u, \quad y=c_{\sigma} x,
$$

for which the switching signal $\sigma$ takes values in the set $\mathcal{P}:=\{1,2\}$ and both unswitched systems have $\mathcal{H}_{\infty}$-norm (and therefore RMS gain) equal to one, i.e.,

$$
\left\|c_{p}\left(s I-A_{p}\right)^{-1} b_{p}\right\|_{\infty}=1, \quad p \in \mathcal{P}
$$

Consider also the switching signal

$$
\sigma(t):=\left\{\begin{array}{ll}
1 & t<1 \\
2 & t \geq 1
\end{array} \quad t \geq 0\right.
$$

Since $\sigma$ has a single discontinuity, it belongs to every family of switching signals $\mathcal{S}\left[\tau_{D}\right], \tau_{D}>0$. Take now the following probe input signal with $\mathcal{L}_{2}$-norm equal to one:

$$
u(t):=\left\{\begin{array}{ll}
1 & t<1 \\
0 & t \geq 1
\end{array} \quad t \geq 0 .\right.
$$

The output of (5) corresponding to the switching signal (7) and the input (8) is given by

$$
y(t)=\left\{\begin{array}{ll}
c_{1}\left(\int_{0}^{t} e^{A_{1}(t-\tau)} d \tau\right) b_{1} & 0 \leq t<1 \\
c_{2} e^{A_{2}(t-1)}\left(\int_{0}^{1} e^{A_{1}(1-\tau)} d \tau\right) b_{1} & t \geq 1
\end{array} \quad t \geq 0 .\right.
$$

It turns out that $c_{2}$ can be chosen arbitrarily large without violating (6), provided that $b_{2}$ is sufficiently small. Therefore, by appropriate choice of $c_{2}$, the $\mathcal{L}_{2}$-norm of $y$ can actually be very 
large (in particular larger than 1) for a unit-norm input, even though we are switching between two systems with RMS gain equal to one. The intuition behind this example is that for $t<1$ the input energy is "stored" in the system through one realization (perhaps through a "large" $b_{1}$ ) and then it is "released" to the output for $t \geq 1$ through a different realization (perhaps through a "large" $c_{2}$ ). From this example, we conclude that even for very slow switching (in the extreme a single switch), the RMS gain of a switched system can be arbitrarily larger than the RMS gains of the "unswitched systems." Moreover, the RMS gain of a switched system is realization dependent and cannot be determined just from the transfer functions of the systems being switched. The goal of this paper is then to compute the slow-switching RMS gain $\mathfrak{g}_{\text {slow }}$ defined by (3).

For linear time-invariant systems, the RMS gain can be determined by solving algebraic Riccati equations. In particular, it is well known (Bounded-real Lemma) that the RMS gain is smaller than a given constant $\gamma>0$ if and only if an algebraic Riccati equation of the form

$$
S(P ; \gamma):=A_{\gamma}^{\prime} P+P A_{\gamma}+P R_{\gamma} P+Q_{\gamma}=0,
$$

has a positive definite solution $P$, where $A_{\gamma}, Q_{\gamma}$, and $R_{\gamma}$ are appropriately defined $\gamma$-dependent $n \times n$ real matrices with $Q_{\gamma}$ and $R_{\gamma}$ symmetric and positive semidefinite (cf. Theorem 1 in Section 3 for details). This allows one to determine the precise value of the RMS gain of a system by using a bisection algorithm to find the smallest value of $\gamma$ for which a positive definite solution to (9) exists [17]. Although in general algebraic Riccati equations have more than one solution, in order to determine the RMS gain of a time-invariant system it is sufficient to find one of these solutions. However, to determine the RMS gain of a switched system, we have to pay special attention to the multiple solutions to the algebraic Riccati equations involved. We shall see below that the condition for the slow-switching RMS gain of a switched system to be smaller than $\gamma$ requires a separation between all the stabilizing and all the antistabilizing solutions to the algebraic Riccati equations of the systems being switched.

The remaining of this paper is organized as follows: In Section 2 we derive a general formula for the RMS gain of a switched system in terms of the solution to a differential Riccati equation. This prompts us to investigate the solutions to differential Riccati equations, which is done in Section 3. In Section 4 we determine upper and lower bounds on the RMS gain of a switched system over the set $\mathcal{S}_{1}$ of switching signals with no more than one discontinuity. We then show in Section 5 that these same bounds are also valid for the slow-switching RMS gain. A bisection-type algorithm to compute it is given in Section 6. Section 7 illustrates the results presented in the context of an example. Section 8 contains final conclusions and directions for future research. A partial version of the results in this paper will be presented at the Fifth Hybrid Systems: Computation and Control Workshop, Stanford, March 2002.

\section{Computation of RMS gains}

To determine the RMS gain of (2) over $\mathcal{S}$ we consider the following optimization problem:

$$
J(\gamma, \sigma):=\lim _{T \rightarrow \infty} \sup _{u \in \mathcal{L}_{2}} \int_{0}^{T}\|y(\tau)\|^{2}-\gamma^{2}\|u(\tau)\|^{2} d \tau, \quad \gamma \geq 0, \sigma \in \mathcal{S},
$$

where $y$ is computed along solutions to $(2)$ with $x(0)=0$. The RMS gain of $(2)$ over $\mathcal{S}$ is then given by

$$
\mathfrak{g}_{\mathcal{S}}:=\inf \{\gamma \geq 0: J(\gamma, \sigma) \leq 0, \forall \sigma \in \mathcal{S}\}
$$


Note that because the system is linear, when $J(\gamma, \sigma)>0$ we must actually have $J(\gamma, \sigma)=+\infty$, which means that $J(\gamma, \sigma) \leq 0$ in (10) could be replaced by $J(\gamma, \sigma)<\infty$. It is also sufficient to consider the case $\gamma>\left\|D_{p}\right\|, p \in \mathcal{P}$ as the RMS gain is always larger or equal to any of the high frequency gains $\left\|D_{p}\right\|$ because of (4).

We shall use dynamic programming to compute $J(\gamma, \sigma)$ for a given $\sigma \in \mathcal{S}$ and $\gamma>\left\|D_{p}\right\|, p \in \mathcal{P}$. To this effect let us define the following finite-horizon "cost-to-go:"

$$
V\left(x_{0}, t ; T, \gamma, \sigma\right):=\sup _{u \in \mathcal{L}_{2}, x(t)=x_{0}} \int_{t}^{T}\|y(\tau)\|^{2}-\gamma^{2}\|u(\tau)\|^{2} d \tau .
$$

The corresponding dynamic-programming equation ${ }^{2}$ is given by ${ }^{3}$

$$
\begin{aligned}
-V_{t}= & \sup _{u \in \mathbb{R}}\left\|C_{\sigma} x_{0}+D_{\sigma} u\right\|^{2}-\gamma^{2}\|u\|^{2}+V_{x}\left(A_{\sigma} x_{0}+B_{\sigma} u\right) \\
=\sup _{u \in \mathbb{R}} V_{x} A_{\sigma} x_{0}+x_{0}^{\prime} C_{\sigma}^{\prime} C_{\sigma} x_{0}+\left(\frac{1}{2} V_{x} B_{\sigma}+x_{0}^{\prime} C_{\sigma}^{\prime} D_{\sigma}\right) X^{-1}\left(\frac{1}{2} B_{\sigma}^{\prime} V_{x}^{\prime}+D_{\sigma}^{\prime} C_{\sigma} x_{0}\right) & \quad-\left(u^{\prime} X-\frac{1}{2} V_{x} B_{\sigma}-x_{0}^{\prime} C_{\sigma}^{\prime} D_{\sigma}\right) X^{-1}\left(X u-\frac{1}{2} B_{\sigma}^{\prime} V_{x}^{\prime}-D_{\sigma}^{\prime} C_{\sigma} x_{0}\right) \\
\quad & \quad+x_{0}^{\prime} C_{\sigma}^{\prime} C_{\sigma} x_{0}+\left(\frac{1}{2} V_{x} B_{\sigma}+x_{0}^{\prime} C_{\sigma}^{\prime} D_{\sigma}\right) X^{-1}\left(\frac{1}{2} B_{\sigma}^{\prime} V_{x}^{\prime}+D_{\sigma}^{\prime} C_{\sigma} x_{0}\right),
\end{aligned}
$$

where $X_{p}:=\gamma^{2} I-D_{p}^{\prime} D_{p}>0$. Since for a fixed $\sigma$ we are dealing with a linear time-varying system, we can restrict our attention to costs-to-go of the form $V\left(x_{0}, t ; T, \gamma, \sigma\right)=x_{0}^{\prime} P(t) x_{0}, t \geq 0$ with $P(t)$ real symmetric. Replacing this in (12) we obtain

$$
0=x_{0}^{\prime}\left(\dot{P}+P A_{\sigma}+A_{\sigma}^{\prime} P+C_{\sigma}^{\prime} C_{\sigma}+\left(P B_{\sigma}+C_{\sigma}^{\prime} D_{\sigma}\right) X_{\sigma}^{-1}\left(B_{\sigma}^{\prime} P+D_{\sigma}^{\prime} C_{\sigma}\right)\right) x_{0}
$$

for every $x_{0}$, which (because of symmetry) actually implies that

$$
-\dot{P}=P A_{\sigma}+A_{\sigma}^{\prime} P+C_{\sigma}^{\prime} C_{\sigma}+\left(P B_{\sigma}+C_{\sigma}^{\prime} D_{\sigma}\right) X_{\sigma}^{-1}\left(B_{\sigma}^{\prime} P+D_{\sigma}^{\prime} C_{\sigma}\right)=S_{\sigma}(P ; \gamma),
$$

where

$$
S_{p}(P ; \gamma):=P \bar{A}_{p}+\bar{A}_{p} P+Q_{p}+P R_{p} P, \quad p \in \mathcal{P},
$$

with $\bar{A}_{p}:=A_{p}+B_{p} X_{p}^{-1} D_{p}^{\prime} C_{p}, R_{p}:=B_{p} X_{p}^{-1} B_{p}^{\prime}, Q_{p}:=C_{p}^{\prime}\left(I+D_{p} X_{p}^{-1} D_{p}^{\prime}\right) C_{p}$. Moreover,

$$
P(T)=0,
$$

\footnotetext{
${ }^{2}$ Equation (11) could be derived as follows: For an elementary interval $d t>0$, we can write

$$
\begin{aligned}
V\left(x_{0}, t ; T, \gamma, \sigma\right) & =\sup _{u \in \mathcal{L}_{2}, x(t)=x_{0}} \int_{t}^{t+d t}\|y(\tau)\|^{2}-\gamma^{2}\|u(\tau)\|^{2} d \tau+\int_{t+d t}^{T}\|y(\tau)\|^{2}-\gamma^{2}\|u(\tau)\|^{2} d \tau \\
& =\sup _{u(t) \in \mathbb{R}}\left(\left\|C_{\sigma(t)} x_{0}+D_{\sigma(t)} u(t)\right\|^{2}-\gamma^{2}\|u(t)\|^{2}\right) d t+V\left(x_{0}+\left(A_{\sigma(t)} x_{0}+B_{\sigma(t)} u(t)\right) d t, t+d t ; T, \gamma, \sigma\right)
\end{aligned}
$$
}

Subtracting $V\left(x_{0}, t+d t ; T, \gamma, \sigma\right)$ to both sides of the above equation, dividing by $d t$ and taking the limit as $d t \rightarrow 0$, we obtain (11).

${ }^{3}$ For simplicity we dropped the dependence of $\left(x_{0}, t ; T, \gamma, \sigma\right)$ on $V$. We denote the partial derivatives of $V$ with respect to $t$ and $x$ by $V_{t}$ and $V_{x}$, respectively. 
since $V\left(x_{0}, T ; T, \gamma, \sigma\right)=0$ for every $x_{0}$. Since (13) is locally Lipschitz with respect to $P$, we conclude that $J(\gamma, \sigma)<\infty$ if and only if the unique solution to the final value problem (13)-(15) exists on every interval $[0, T), T>0$ (cf. Appendix for details). Therefore

$$
\mathfrak{g}_{\mathcal{S}}=\inf \{\gamma \geq 0: \text { solution to }(13)-(15) \text { exists on }[0, T), \forall T>0, \sigma \in \mathcal{S}\} .
$$

To compute the RMS gain of a switched system we must then study the existence of solution to a differential Riccati equation. We do this in the following section.

\section{Differential Riccati equations}

We start by considering the following algebraic Riccati equation

$$
S(P):=A^{\prime} P+P A+P R P+Q=0,
$$

where $A, Q$, and $R$ are $n \times n$ real matrices with $Q$ and $R$ symmetric. Associated with this equation one defines a $2 n \times 2 n$ Hamiltonian matrix by

$$
H:=\left[\begin{array}{cc}
A & R \\
-Q & -A^{\prime}
\end{array}\right]
$$

In the sequel, we say that a matrix $P^{-}$is a stabilizing solution to the algebraic Riccati equation (17) if $P^{-}$is real, symmetric, positive definite, and $A+R P^{-}$is Hurwitz. We say that $P^{+}$is an antistabilizing solution to (17) if $P_{+}$is real, symmetric, positive definite, and $-\left(A+R P^{+}\right)$is Hurwitz. The following theorem is a consequence of several well known results on algebraic Riccati equations. For completeness we include in the Appendix the main steps needed to prove it.

Theorem 1. Consider a minimal realization $(\bar{A}, \bar{B}, \bar{C}, \bar{D})$ with $\bar{A}$ Hurwitz and

$$
\left\|\bar{C}(s I-\bar{A})^{-1} \bar{B}+\bar{D}\right\|_{\infty}<\gamma
$$

for some $\gamma>0$. Then $\|D\|<\gamma$ and there exist stabilizing and antistabilizing solutions $P^{-}$and $P^{+}$, respectively, to the algebraic Riccati equation (17) with

$$
\begin{array}{ll}
A:=\bar{A}+\bar{B} \bar{X}^{-1} \bar{D}^{\prime} \bar{C}, & R:=\bar{B} \bar{X}^{-1} \bar{B}^{\prime}, \\
Q:=\bar{C}^{\prime}\left(I+\bar{D} \bar{X}^{-1} \bar{D}^{\prime}\right) \bar{C}, & \bar{X}:=\gamma^{2} I-\bar{D}^{\prime} \bar{D} .
\end{array}
$$

Moreover, $P_{+}-P_{-}>0{ }^{4}$ and these solutions can be computed by

$$
P^{-}=P_{2}^{-}\left(P_{1}^{-}\right)^{-1}, \quad P^{+}=P_{2}^{+}\left(P_{1}^{+}\right)^{-1},
$$

where the columns of $\left[{P_{1}^{-\prime}}^{P_{2}^{-\prime}}\right]^{\prime}$ and $\left[{{ }_{1}^{+^{\prime}}}^{{ }^{+{ }^{+}}}\right]^{\prime}$ are chosen to form bases for the stable and antistable invariant $t^{5}$ subspaces of $H$, respectively. The matrices $P_{1}^{-}$and $P_{1}^{+}$above are guaranteed to be nonsingular.

\footnotetext{
${ }^{4}$ Given a symmetric matrix $Q$ we write $Q>0, Q \geq 0, Q<0$, and $Q \leq 0$ when $Q$ is positive definite, positive semidefinite, negative definite, and negative semidefinite, respectively. We write $Q \nsupseteq 0$ when $Q$ is nonsingular and not positive semidefinite (therefore $Q$ must have at least one strictly negative eigenvalue). Similarly, $Q \not \leq 0$ means that $Q$ is nonsingular and not negative semidefinite.

${ }^{5}$ Let $H$ be a matrix with characteristic polynomial $\alpha(s)=\alpha^{-}(s) \alpha^{0}(s) \alpha^{+}(s)$, where all roots of the polynomials $\alpha^{-}(s), \alpha^{+}(s)$, and $\alpha^{0}(s)$ have negative, positive, and zero real parts, respectively. The stable and antistable invariant subspaces of $H$ are defined to be $\operatorname{Ker} \alpha^{-}(H)$ and $\operatorname{Ker} \alpha^{+}(H)$, respectively.
} 
Consider now the final-value solution to the following differential Riccati equation

$$
-\dot{P}=S(P)=A^{\prime} P+P A+P R P+Q, \quad P\left(t_{0}\right)=P_{0}, \quad t \leq t_{0} .
$$

The following theorem is inspired by results from [19] and [20, Chapter 3].

Theorem 2. Suppose that there exist stabilizing and antistabilizing solutions to (17) with $P^{+}-$ $P^{-}>0$.

(i) When $P_{0}-P^{+}$is nonsingular, the solution $\Pi\left(t ; P_{0}\right)$ to $(20)$ is given by

$$
\Pi\left(t ; P_{0}\right)=P^{+}+\Lambda(t)^{-1}, \quad t \leq t_{0},
$$

with

$$
\Lambda(t):=e^{\left(A+R P^{+}\right)\left(t-t_{0}\right)}\left(\left(P_{0}-P^{+}\right)^{-1}+\left(P^{+}-P^{-}\right)^{-1}\right) e^{\left(A+R P^{+}\right)^{\prime}\left(t-t_{0}\right)}-\left(P^{+}-P^{-}\right)^{-1}
$$

on the interval $\mathcal{I}$ on which $\Lambda$ is nonsingular.

(ii) When $R \geq 0$ and $P_{0}-P^{+}<0$ the solution $\Pi\left(t ; P_{0}\right)$ to $(20)$ exists globally for $t \leq t_{0}$ and $\Pi\left(t ; P_{0}\right) \rightarrow P^{-}$as $t \rightarrow-\infty$. The same result holds when $R \leq 0$ and $P_{0}-P^{+}>0$.

(iii) When $R \geq 0$ and $P_{0}-P^{+} \not \leq 0$, the solution $\Pi\left(t ; P_{0}\right)$ to (20) has finite escape time. The same result holds when $R \leq 0$ and $P_{0}-P^{+} \nsupseteq 0$.

Proof of Theorem 2. To prove (i), let $P:=P^{+}+\Lambda^{-1}, t \in \mathcal{I}$. Since $\left(P-P^{+}\right) \Lambda=I$, we conclude that $\dot{P} \Lambda+\left(P-P^{+}\right) \dot{\Lambda}=0$. On the other hand, differentiating (21), we obtain

$$
\dot{\Lambda}=\left(A+R P^{+}\right)\left(\Lambda+\left(P^{+}-P^{-}\right)^{-1}\right)+\left(\Lambda+\left(P^{+}-P^{-}\right)^{-1}\right)\left(A+R P^{+}\right)^{\prime} .
$$

Therefore,

$$
\dot{P} \Lambda+\left(P-P^{+}\right)\left(A+R P^{+}\right)\left(\Lambda+\left(P^{+}-P^{-}\right)^{-1}\right)+\left(P-P^{+}\right)\left(\Lambda+\left(P^{+}-P^{-}\right)^{-1}\right)\left(A+R P^{+}\right)^{\prime}=0,
$$

which is further equivalent to

$$
\begin{aligned}
-\dot{P}=(P- & \left.P^{+}\right)\left(A+R P^{+}\right)+\left(A+R P^{+}\right)^{\prime}\left(P-P^{+}\right) \\
& +\left(P-P^{+}\right)\left(\left(A+R P^{+}\right)\left(P^{+}-P^{-}\right)^{-1}+\left(P^{+}-P^{-}\right)^{-1}\left(A+R P^{+}\right)^{\prime}\right)\left(P-P^{+}\right),
\end{aligned}
$$

because $\Lambda^{-1}=P-P^{+}$. Moreover, since

$$
\begin{aligned}
0 & =S\left(P^{+}\right)-S\left(P^{-}\right)=A^{\prime}\left(P^{+}-P^{-}\right)+\left(P^{+}-P^{-}\right) A+P^{+} R P^{+}-P^{-} R P^{-} \\
& =\left(A+R P^{+}\right)^{\prime}\left(P^{+}-P^{-}\right)+\left(P^{+}-P^{-}\right)\left(A+R P^{+}\right)-\left(P^{+}-P^{-}\right) R\left(P^{+}-P^{-}\right),
\end{aligned}
$$

we conclude that

$$
\left(P^{+}-P^{-}\right)^{-1}\left(A+R P^{+}\right)^{\prime}+\left(A+R P^{+}\right)\left(P^{+}-P^{-}\right)^{-1}-R=0 .
$$


From this and (22), we obtain

$$
\begin{aligned}
-\dot{P} & =\left(P-P^{+}\right)\left(A+R P^{+}\right)+\left(A+R P^{+}\right)^{\prime}\left(P-P^{+}\right)+\left(P-P^{+}\right) R\left(P-P^{+}\right) \\
& =P A+A^{\prime} P+P R P-P^{+} A-A^{\prime} P^{+}-P^{+} R P^{+} \\
& =P A+A^{\prime} P+P R P+Q-S\left(P^{+}\right)
\end{aligned}
$$

Since $S\left(P^{+}\right)=0$, we conclude that $P$ satisfies the differential equation in (20). Moreover, because $\Lambda\left(t_{0}\right)=\left(P_{0}-P^{+}\right)^{-1}$, we conclude that $P\left(t_{0}\right)=P_{0}$ and therefore $P^{+}+\Lambda^{-1}$ is indeed the solution to $(20)$ on $\mathcal{I}$.

To prove (ii), let

$$
\Gamma(t):=e^{\left(A+R P^{+}\right)\left(t-t_{0}\right)}\left(P^{+}-P^{-}\right)^{-1} e^{\left(A+R P^{+}\right)^{\prime}\left(t-t_{0}\right)}-\left(P^{+}-P^{-}\right)^{-1} .
$$

Differentiating this equation, we conclude that $\Gamma$ is the solution to the following differential Lyapunov equation

$$
\begin{aligned}
\dot{\Gamma} & =\left(A+R P^{+}\right)\left(\Gamma+\left(P^{+}-P^{-}\right)^{-1}\right)+\left(\Gamma+\left(P^{+}-P^{-}\right)^{-1}\right)\left(A+R P^{+}\right)^{\prime} \\
& =\left(A+R P^{+}\right) \Gamma+\Gamma\left(A+R P^{+}\right)^{\prime}+R
\end{aligned}
$$

with initial condition $\Gamma\left(t_{0}\right)=0$. Here, we used (23). Since, for $R \geq 0$,

$$
\Gamma(t)=\int_{t_{0}}^{t} e^{\left(A+R P^{+}\right)(t-\tau)} R e^{\left(A+R P^{+}\right)^{\prime}(t-\tau)} d \tau \leq 0, \quad t \leq t_{0},
$$

we conclude that

$$
\begin{aligned}
\Lambda(t) & =e^{\left(A+R P^{+}\right)\left(t-t_{0}\right)}\left(P_{0}-P^{+}\right)^{-1} e^{\left(A+R P^{+}\right)^{\prime}\left(t-t_{0}\right)}+\Gamma(t) & \\
& \leq e^{\left(A+R P^{+}\right)\left(t-t_{0}\right)}\left(P_{0}-P^{+}\right)^{-1} e^{\left(A+R P^{+}\right)^{\prime}\left(t-t_{0}\right)}, & t \leq t_{0} .
\end{aligned}
$$

Since $e^{\left(A+R P^{+}\right)\left(t-t_{0}\right)}$ is nonsingular for any finite $t$, we conclude that $\Lambda(t)<0, \forall t \leq t_{0}$ and therefore $\mathcal{I}=\left(-\infty, t_{0}\right]$ whenever $P_{0}-P^{+}<0$. In this case, and because $-\left(A+R P^{+}\right)$is Hurwitz, $e^{\left(A+R P^{+}\right)\left(t-t_{0}\right)} \rightarrow 0$ as $t \rightarrow-\infty$. Therefore $\Lambda(t) \rightarrow-\left(P^{+}-P^{-}\right)^{-1}$ and $P(t) \rightarrow P^{-}$as $t \rightarrow-\infty$. Note that for $R \leq 0$, we have $\Gamma(t) \geq 0, \forall t \leq t_{0}$. In this case $P_{0}-P^{+}>0$ guarantees that $\Lambda(t)$ remains positive definite for $t \leq t_{0}$, also leading to global existence of solution.

To prove (iii) note that because $-\left(A+R P^{+}\right)$is a stability matrix, $\Gamma(t)$ above converges to a negative definite matrix as $t \rightarrow-\infty$ and, since $\Lambda$ and $\Gamma$ only differ by a term that converges to zero as $t \rightarrow-\infty, \Lambda(t)$ must also converge to a negative definite matrix. Suppose now that $\Lambda\left(t_{0}\right)=\left(P_{0}-P^{+}\right)^{-1} \not \leq 0$ and therefore that $\Lambda\left(t_{0}\right)$ must have at least one positive eigenvalue. Since as $t \rightarrow \infty$ all eigenvalues of $\Lambda(t)$ must become negative and the eigenvalues are continuous functions of $t, \Lambda(t)$ must be singular for some finite time resulting in finite escape time for the solution $\Pi\left(t ; P_{0}\right)=P^{+}+\Lambda^{-1}(t)$. A similar argument could be used for the case $R \leq 0$ and $P_{0}-P^{+} \nsupseteq 0$.

\section{RMS gain of single-switch systems}

In this section we consider the set $\mathcal{S}_{1}$ of switching signals with no more than one discontinuity. Using the results from the previous section we compute bounds on the RMS gain $\mathfrak{g}_{\mathcal{S}_{1}}$ of (2) over $\mathcal{S}_{1}$ : 
Theorem 3. Assume that the realizations in (1) are minimal. Given any $\gamma>\mathfrak{g}_{\text {static }}$,

$$
\begin{aligned}
& \exists p, q \in \mathcal{P} \quad P_{q}^{+}-P_{p}^{-} \geq 0 \quad \Rightarrow \quad \mathfrak{g}_{\mathcal{S}_{1}} \geq \gamma \\
& \forall p, q \in \mathcal{P} \quad P_{q}^{+}-P_{p}^{-}>0 \quad \Rightarrow \quad \mathfrak{g}_{\mathcal{S}_{1}} \leq \gamma,
\end{aligned}
$$

where $P_{p}^{+}$and $P_{p}^{-}, p \in \mathcal{P}$ respectively denote the stabilizing and antistabilizing solutions to the algebraic Riccati equation $S_{p}(P ; \gamma)=0$, with $S_{p}$ defined by (14).

Before proving Theorem 3, it is interesting to note that the condition in (26) that gives the upper bound on the RMS gain requires a separation between all the stabilizing and all the antistabilizing solutions to the algebraic Riccati equations of the systems being switched. Note that for $p=q$, the fact that the $q$ th system has RMS gain smaller than $\gamma$ already guarantees that $P_{q}^{+}-P_{q}^{-}>0$ (cf. Theorem 1). However, this is not enough for $\mathfrak{g}_{\mathcal{S}_{1}}$ to be smaller or equal to $\gamma$. Indeed, we need all the stabilizing solutions $P_{p}^{-}$to be "smaller" than all the antistabilizing solutions $P_{q}^{+}$.

Proof of Theorem 3. To prove (25) we show that when $P_{q}^{+}-P_{p}^{-} \nsucceq 0$ for some $p, q \in \mathcal{P}$, the RMS gain $\mathfrak{g}_{\mathcal{S}_{1}}$ given by (16) must be larger or equal to $\gamma$ because the solution to (13)-(15) does not exist for some $T>0, \sigma \in \mathcal{S}$. To this effect suppose that there are $p, q \in \mathcal{P}$ for which $P_{q}^{+}-P_{p}^{-} \geq 0$ and take

$$
\sigma(t):=\left\{\begin{array}{ll}
q & t<T_{\text {switch }} \\
p & t \geq T_{\text {switch }}
\end{array} \quad t \geq 0,\right.
$$

for some $T_{\text {switch }}>0$. Because $R_{p} \geq 0$ and $-P_{p}^{+}<0$ (cf. Theorem 1), we conclude from Theorem 2 (ii) that the solution to (13)-(15) exists for every $t \in\left[T_{\text {switch }}, T\right)$. Moreover, if $\sigma$ where equal to $p$ for all times, we would actually have $P(t) \rightarrow P_{p}^{-}$as $t \rightarrow-\infty$. This means that by choosing $T$ sufficiently large, it is possible to have $P\left(T_{\text {switch }}\right)$ sufficiently close to $P_{p}^{-}$so that $P_{q}^{+}-P\left(T_{\text {switch }}\right) \nsupseteq 0$. Here, we are using the fact that the set $\left\{P: P_{q}^{+}-P \nsupseteq 0\right\}$ is open and therefore if $P\left(T_{\text {switch }}\right)$ is sufficiently close to the element $P_{p}^{-}$of this set, it must be inside the set. Once it is known that $T$ is chosen such that $P_{q}^{+}-P\left(T_{\text {switch }}\right) \nsupseteq 0$, by applying Theorem 2 to solve (13)-(15) for $t \leq T_{\text {switch }}$, we conclude from (iii) that the solution is actually only defined in an interval $\left(T_{\text {escape }}, T_{\text {switch }}\right]$ of finite length. Because of time invariance, we can choose $T$ sufficiently large so that $T_{\text {escape }}>0$. So the solution to (13)-(15) does not exist globally. This finishes the proof of (25).

To prove (26) we show directly that when $P_{q}^{+}-P_{p}^{-}>0$ for every $p, q \in \mathcal{P}$, we have $\|y\|_{2} \leq \gamma\|u\|_{2}$ for every input and switching signals. To this effect pick arbitrary $u \in \mathcal{L}_{2}, \sigma \in \mathcal{S}_{1}$ and define

$$
v(t):=V(x(t), t)+\int_{0}^{t}\|y(\tau)\|^{2}-\gamma^{2}\|u(\tau)\|^{2} d \tau, \quad t \geq 0
$$

where $x$ and $y$ are evaluated along a solution to $(2)$ and $V(x, t):=x^{\prime} P(t) x, t \geq 0$. Here, $P(t)$, $t \in[0, \infty)$ denotes a time-varying continuous real symmetric matrix with the property that on any interval on which $\sigma$ is constant we have

$$
-\dot{P}=S_{\sigma}(P ; \gamma)
$$

To construct such $P(t)$ we use the fact that because $\sigma \in \mathcal{S}_{1}$, the switching signal must be of the form (27) (possibly with $p=q$ if it is actually constant). Suppose then that for $t>T_{\text {switch }}$ we set $P(t)$ constant and equal to $P_{p}^{-}$, and for $t \leq T_{\text {switch }} P(t)$ is defined by the final value problem

$$
-\dot{P}=S_{q}(P ; \gamma), \quad P\left(T_{\text {switch }}\right)=P_{p}^{-} .
$$


The function $P(t)$ is well defined on $t \in\left[0, T_{\text {switch }}\right]$ because $P_{p}^{-}-P_{q}^{+}<0$ (cf. Theorem 2 (ii)) and also on $t \in\left(T_{\text {switch }},+\infty\right)$ because we are simply setting it to be equal to $P_{p}^{-}$on this interval. By construction this choice of $P(t)$ is continuous and does satisfy (28) on every interval on which $\sigma$ is constant. Because of (28), on any such interval we have

$$
\begin{aligned}
\dot{v}=x^{\prime} \dot{P} x+ & V_{x}\left(A_{\sigma} x+B_{\sigma} u\right)+\left\|C_{\sigma} x+D_{\sigma} u\right\|^{2}-\gamma^{2}\|u\|^{2} \\
=x^{\prime} \dot{P} x+ & V_{x} A_{\sigma} x_{0}+x_{0}^{\prime} C_{\sigma}^{\prime} C_{\sigma} x_{0}+ \\
& +\left(\frac{1}{2} V_{x} B_{\sigma}+x_{0}^{\prime} C_{\sigma}^{\prime} D_{\sigma}\right) X^{-1}\left(\frac{1}{2} B_{\sigma}^{\prime} V_{x}^{\prime}+D_{\sigma}^{\prime} C_{\sigma} x_{0}\right) \\
& \quad-\left(u^{\prime} X-\frac{1}{2} V_{x} B_{\sigma}-x_{0}^{\prime} C_{\sigma}^{\prime} D_{\sigma}\right) X^{-1}\left(X u-\frac{1}{2} B_{\sigma}^{\prime} V_{x}^{\prime}-D_{\sigma}^{\prime} C_{\sigma} x_{0}\right) \\
\leq x^{\prime} \dot{P} x+ & V_{x} A_{\sigma} x_{0}+x_{0}^{\prime} C_{\sigma}^{\prime} C_{\sigma} x_{0}+ \\
& \quad+\left(\frac{1}{2} V_{x} B_{\sigma}+x_{0}^{\prime} C_{\sigma}^{\prime} D_{\sigma}\right) X^{-1}\left(\frac{1}{2} B_{\sigma}^{\prime} V_{x}^{\prime}+D_{\sigma}^{\prime} C_{\sigma} x_{0}\right), \\
=x^{\prime}(\dot{P}+ & \left.S_{\sigma}(P ; \gamma)\right) x=0 .
\end{aligned}
$$

(cf. equations (12)-(13)). We then conclude that $v$ decreases between discontinuities of $\sigma$ and is continuous at the switching time $T_{\text {switch. Therefore }} v(t) \leq v(0)=x(0)^{\prime} P(0) x(0), \forall t \geq 0$. For a zero initialization of $(2)$ we then conclude that $v(t) \leq 0, \forall t \geq 0$, or equivalently that

$$
\int_{0}^{t}\|y(\tau)\|^{2}-\gamma^{2}\|u(\tau)\|^{2} d \tau \leq-V(x(t), t), \quad \forall t \geq 0 .
$$

Since $u \in \mathcal{L}_{2}, \lim _{t \rightarrow \infty} x(t)=0$ and therefore $\int_{0}^{\infty}\|y(\tau)\|^{2}-\gamma^{2}\|u(\tau)\|^{2} d \tau \leq 0$. This concludes the proof since it shows that indeed $\|y\|_{2} \leq \gamma\|u\|_{2}$.

\section{$5 \quad$ Slow-switching RMS gain}

In this section we show that the upper and lower bounds provided by Theorem 3 on the RMS gain $\mathfrak{g}_{\mathcal{S}_{1}}$ of (2) over $\mathcal{S}_{1}$ also hold for the slow-switching RMS gain $\mathfrak{g}_{\text {slow }}$ of (2). For simplicity, in this section we assume that the set $\mathcal{P}$ is finite, i.e., that we are switching among a finite number of systems. However, it would be straightforward to replace this assumption by uniformity conditions on the systems being switched, e.g., compactness of the family of realizations $\left\{A_{p}, B_{p}, C_{p}, D_{p}: p \in\right.$ $\mathcal{P}\}$.

Theorem 4. Assume that the realizations in (1) are minimal and $\mathcal{P}$ is finite. Given any $\gamma>\mathfrak{g}_{\text {static }}$

$$
\begin{array}{llll}
\exists p, q \in \mathcal{P} & P_{q}^{+}-P_{p}^{-} \not 0 & \Rightarrow & \mathfrak{g}_{\text {slow }} \geq \gamma \\
\forall p, q \in \mathcal{P} & P_{q}^{+}-P_{p}^{-}>0 & \Rightarrow & \mathfrak{g}_{\text {slow }} \leq \gamma,
\end{array}
$$

where $P_{p}^{+}$and $P_{p}^{-}, p \in \mathcal{P}$ respectively denote the stabilizing and antistabilizing solutions to the algebraic Riccati equation $S_{p}(P ; \gamma)=0$, with $S_{p}$ defined by $(14)$.

Proof of Theorem 4. To prove (29) note that for any $\tau_{D}>0, \mathcal{S}_{1} \subset \mathcal{S}\left[\tau_{D}\right]$ and therefore $\mathfrak{g}_{\mathcal{S}_{1}} \leq \mathfrak{g}_{\text {slow }}$. From Theorem 3 we then conclude that

$$
\exists p, q \in \mathcal{P} \quad P_{q}^{+}-P_{p}^{-} \nsupseteq 0 \quad \Rightarrow \quad \mathfrak{g}_{\mathcal{S}_{1}} \geq \gamma \quad \Rightarrow \quad \mathfrak{g}_{\text {slow }} \geq \mathfrak{g}_{\mathcal{S}_{1}} \geq \gamma .
$$


To prove (30) assume that $P_{q}^{+}-P_{p}^{-}>0$ for every $p, q \in \mathcal{P}$ and therefore that we can select a positive constant $\epsilon$ and matrices $P_{(p, q)}, p, q \in \mathcal{P}$ such that

$$
\left\|P-P_{p}^{-}\right\| \leq \epsilon \quad \Rightarrow \quad P_{q}^{+}>P_{(p, q)} \geq P, \quad \forall p, q \in \mathcal{P} .
$$

In practice, $P_{(p, q)}$ can be any matrix sufficiently close to $P_{p}^{-}$so that $P_{q}^{+}>P_{(p, q)}>P_{p}^{-}$and $\epsilon$ a sufficiently small positive constant so that for any $P$ such that $\left\|P-P_{p}^{-}\right\| \leq \epsilon$ we still have $P_{(p, q)} \geq P$. Since $P_{(p, q)}-P_{q}^{+}<0$, we conclude from Theorem 2 (ii) that the solution to the final value problem

$$
-\dot{P}=S_{q}(P ; \gamma), \quad P(T)=P_{(p, q)},
$$

exists globally and converges to $P_{q}^{-}$as $t \rightarrow-\infty$. Pick now a constant $\tau_{D}$ sufficiently large so that $\left\|P(t)-P_{q}^{-}\right\| \leq \epsilon, \forall t \leq T-\tau_{D}$ regardless of the choices for $p$ and $q$ in (32). We show next that the RMS gain over $\mathcal{S}\left[\tau_{D}\right]$ is smaller or equal to $\gamma$ and therefore so is the slow-switching RMS gain $\mathfrak{g}_{\text {slow }}$.

Pick arbitrary $u \in \mathcal{L}_{2}, \sigma \in \mathcal{S}_{1}$ and define

$$
v(t):=V(x(t), t)+\int_{0}^{t}\|y(\tau)\|^{2}-\gamma^{2}\|u(\tau)\|^{2} d \tau, \quad t \geq 0,
$$

where $x$ and $y$ are evaluated along a solution to $(2)$ and $V(x, t):=x^{\prime} P(t) x, t \geq 0$. Here, $P(t)$ denotes a time-varying symmetric matrix with the property that on any interval $\left(t_{1}, t_{2}\right)$ on which $\sigma$ is constant and equal to $q$ we have

$$
-\dot{P}=S_{q}(P ; \gamma), \quad P\left(t_{2}\right)=P_{0},
$$

where $P_{0}$ is chosen equal to $P_{q}^{-}$in case $t_{2}=\infty$ and equal to $P_{(p, q)}$ when $t_{2}<\infty$, with $p$ being the value of $\sigma$ immediately after $t_{2}$. Note that in the former case (which occurs only when $t_{1}$ is the last switching time), $P(t)$ is constant for $t>t_{1}$. In the latter case, the solution to (33) is well defined for every $t \leq t_{2}$ because $P_{(p, q)}-P_{q}^{+}<0$ (cf. Theorem 2 (ii)). This construction yields a time-varying matrix $P(t)$ that is well defined for all $t \in(0, \infty)$ and satisfies $(28)$ on every interval on which $\sigma$ is constant. However, now $P(t)$ is not necessarily a continuous function of $t$.

As in the proof of Theorem 3, we conclude that $\dot{v} \leq 0$ on any interval on which $\sigma$ is constant but now $v(t)$ is discontinuous at switching times. In particular, if $\sigma$ switches from $q$ to $p$ at time $t_{2}$, we have

$$
\lim _{\tau \downarrow t_{2}} v(\tau)-\lim _{\tau \uparrow t_{2}} v(\tau)=x\left(t_{2}\right)^{\prime}\left(\bar{P}-P_{p, q}\right) x\left(t_{2}\right),
$$

where $\bar{P}$ is either equal to $P_{p}^{-}$in case $t_{2}$ is the last switching time or the solution at time $t_{2}$ of a final value problem of the form

$$
-\dot{P}=S_{p}(P ; \gamma), \quad P\left(t_{3}\right)=P_{(\bar{p}, p)},
$$

for some $\bar{p} \in \mathcal{P}$ and with $t_{3} \geq t_{2}+\tau_{D}$. However, we selected $\tau_{D}$ sufficiently large so that $\| P\left(t_{2}\right)-$ $P_{p}^{-} \| \leq \epsilon$. Therefore we always have $\left\|\bar{P}-P_{p}^{-}\right\| \leq \epsilon$ whether or not $t_{2}$ is the last switching time. From this and (31) we conclude that $\bar{P}-P_{p, q} \leq 0$ and therefore

$$
\lim _{\tau \downarrow t_{2}} v(\tau)-\lim _{\tau \uparrow t_{2}} v(\tau)=x\left(t_{2}\right)^{\prime}\left(\bar{P}-P_{p, q}\right) x\left(t_{2}\right) \leq 0 .
$$


This means that even at points of discontinuity of $v$, this signal decreases and therefore also here $v(t) \leq v(0), \forall t \geq 0$. As in the proof of Theorem 3 we can then conclude that $\|y\|_{2} \leq \gamma\|u\|_{2}$ for a zero initialization of (2). This finishes the proof since we showed that the RMS gain over $\mathcal{S}\left[\tau_{D}\right]$ is indeed no larger than $\gamma$.

Remark 1. The proof of (30) actually provides a bound on the dwell-time $\tau_{D}$ to have the RMS gain over $\mathcal{S}\left[\tau_{D}\right]$ smaller or equal to $\gamma$. In general, if the separation between the stabilizing and antistabilizing is large, we can choose the $\epsilon$ in (31) large and therefore have an RMS gain no larger than $\gamma$ even for small $\tau_{D}$.

\section{Computation of the slow-switching RMS gain}

Theorem 4 suggests the bisection algorithm in Table 1 to compute the slow-switching RMS gain $\mathfrak{g}_{\text {slow }}$ of (2). This algorithm only fails if there is a positive-length interval of values for $\gamma$ on which

1. Select a tolerance $\epsilon$, an upper bound $\gamma_{\text {up }}$ and a lower bound $\gamma_{\text {low }}$ on $\mathfrak{g}_{\text {slow }}$ (e.g., $\gamma_{\text {low }}:=\mathfrak{g}_{\text {static }}$ and $\gamma_{\text {up }}$ taken from [12])

2. If $\gamma_{\text {up }}-\gamma_{\text {low }} \leq 2 \epsilon$ then stop (the $R M S$ gain $\mathfrak{g}_{\text {slow }}$ is away from $\frac{\gamma_{\text {up }}+\gamma_{\text {low }}}{2}$ by less than $\epsilon$ )

3. Otherwise set $\gamma=\frac{\gamma_{\text {up }}+\gamma_{\text {low }}}{2}$ and

$$
\begin{aligned}
& \text { If } \exists p, q \in \mathcal{P} \quad P_{q}^{+}-P_{p}^{-} \nsucceq 0 \quad \text { then set } \quad \gamma_{\text {low }}=\gamma \\
& \text { If } \forall p, q \in \mathcal{P} \quad P_{q}^{+}-P_{p}^{-}>0 \text { then set } \gamma_{\text {up }}=\gamma
\end{aligned}
$$

and go back to step 2

Table 1: Bisection algorithm to compute the slow-switching RMS gain

all $P_{q}^{+}-P_{p}^{-} \geq 0$ and at least one of these matrices always has an eigenvalue exactly at the origin. Generically, this should not happen over an interval of positive length since an infinitely small perturbation on $\gamma$ should make the eigenvalue become either positive or negative. However, even when this happens one can still use algorithms similar to the one in Table 1 to compute the following upper and lower bounds on $\mathfrak{g}_{\text {slow }}$ :

$$
\sup \left\{\gamma>\mathfrak{g}_{\text {static }}: \exists p, q \in \mathcal{P}, P_{q}^{+}-P_{p}^{-} \geq 0\right\} \leq \mathfrak{g}_{\text {slow }} \leq \inf \left\{\gamma>\mathfrak{g}_{\text {static }}: P_{q}^{+}-P_{p}^{-}>0, \forall p, q \in \mathcal{P}\right\} .
$$

Remark 2. The verification of the matrix-inequality conditions in step 3 is computationally simple when $\mathcal{P}$ is a finite set. Indeed, when $\mathcal{P}$ has $n$ elements, in the worst case, the verification of this conditions requires the computation of the stabilizing and anti-stabilizing solutions to $n$ algebraic Riccati equations (e.g., using (19) in Theorem 1 ) and then verifying if $n(n-1)$ matrices are positive definite. However, if $\mathcal{P}$ is a continuum (i.e., when one can switch among an infinitely large family of systems) the above algorithm may not be practical.

\section{$7 \quad$ Example}

In this section we illustrate the results presented above in the context of a simple example. We consider here the control of the roll angle of an aircraft and use the following process model taken 
from $[21$, p. 381]:

$$
H(s)=\frac{-1000}{s(s+.875)(s+50)} .
$$

For this process we designed two feedback controllers to be connected to the process as in Figure 1. In this figure $u$ denotes the control input, $y$ the process output, $r$ a reference signal, $d$ an input disturbance, and $n$ measurement noise. The two controllers designed essentially differ by the

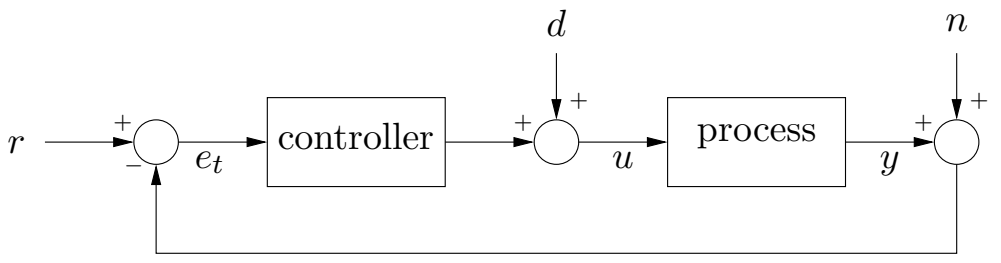

Figure 1: Feedback configuration

closed-loop bandwidth that they achieve: Controller $K_{1}$ exhibits small closed-loop bandwidth and is therefore not very sensitive to noise but exhibits a slow response, whereas controller $K_{2}$ has larger bandwidth and is therefore faster but more sensitive to noise (cf. Figure 2). Both controllers were designed using LQG/LQR. We computed the regulator gains by minimizing the cost

$$
J_{\text {reg }}:=\int_{0}^{\infty} y^{2}(\tau)+\dot{y}^{2}(\tau)+\rho u^{2}(\tau) d \tau
$$

where $\rho$ was chosen equal to 10 and .1 for $K_{1}$ and $K_{2}$, respectively. The design of the optimal LQG gain was done assuming that the input disturbance $d$ and the measurement noise $n$ were uncorrelated white noise processes with $\mathrm{E}[d(t) d(\tau)]=\delta(t-\tau), \mathrm{E}[n(t) n(\tau)]=\mu \delta(t-\tau)$, where $\mu$ was chosen equal to $10^{-1}$ and $10^{-3}$ for $K_{1}$ and $K_{2}$, respectively. The controller transfer functions obtained were:

$$
K_{1}(s) \approx \frac{-20.73(s+50.03)(s+0.9644)}{(s+49.85)\left(s^{2}+17.45 s+144.3\right)}, \quad K_{2}(s) \approx \frac{-1980 .(s+51.01)(s+0.9901)}{(s+57.17)\left(s^{2}+71.28 s+2925\right)} .
$$

Although both controllers exhibit different bandwidths (cf. Figure 2), their closed-loop RMS gains from the noise $n$ to the output $y$ are relatively similar: $1.008(.069 \mathrm{~dB})$ and 1.01 (.087dB) for controllers $K_{1}$ and $K_{2}$, respectively.

Suppose now that we implement the following multi-controller [1] to switch between the two controllers :

$$
\dot{z}=F_{\sigma} z+g_{\sigma} e_{t}, \quad u=h_{\sigma} z,
$$

where each $\left\{F_{p}, g_{p}, h_{p}\right\}, p \in\{1,2\}$ denotes a balanced realization of the controller transfer function $K_{p}(s)$, and $\sigma:[0, \infty) \rightarrow\{1,2\}$ a switching signal that effectively determines which controller is connected to the process at each instant of time. The closed-loop system is then a switched system of the form

$$
\dot{x}=A_{\sigma} x+b_{\sigma}(r-n)+f d, \quad e_{t}=c_{\sigma} x+r-n,
$$




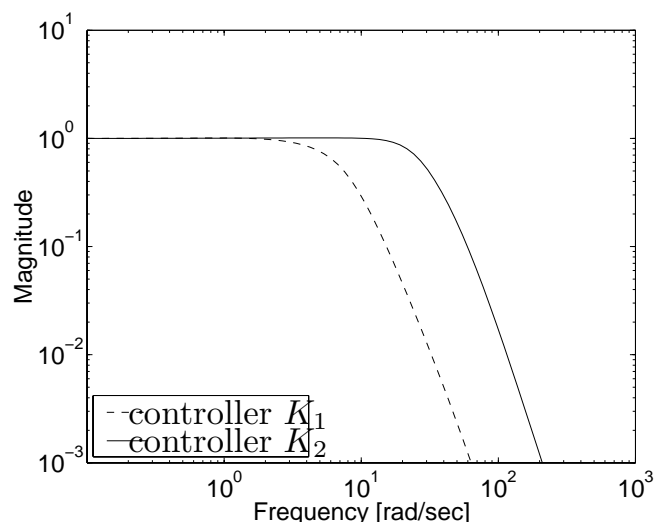

Figure 2: Magnitude Bode plots for the closed-loop transfer functions from the noise $n$ to the output $y$, for the two time-invariant controllers.

where

$$
A_{p}:=\left[\begin{array}{cc}
A & b h_{p} \\
-g_{p} c & F_{p}
\end{array}\right], \quad b_{p}:=\left[\begin{array}{c}
0 \\
g_{p}
\end{array}\right], \quad c_{p}:=\left[\begin{array}{cc}
-c & 0
\end{array}\right], \quad f:=\left[\begin{array}{l}
b \\
0
\end{array}\right], \quad p \in\{1,2\},
$$

and $\{A, b, c\}$ denotes a minimal realization of the process transfer matrix $H(s)$. Using the algorithm in Section 6, we obtained for (34) a slow-switching RMS gain from the noise $n$ to the output $y$ of $9.04(19.1 \mathrm{~dB})$. This example shows that noise entering the system can be greatly amplified because of switching. Note that the RMS gain depends on the realizations chosen for the controllers. Here, we chose balanced realizations because they are generally believed to be well conditioned and, in fact, other realizations tried led to larger RMS gains. An interesting question that remains open is the design of controllers and the choice of realizations that minimize the slow-switching RMS gain. The study of controller realizations that are adequate for switching was pursued in [22]. However, this problem was only studied from a stability perspective and the effect of controllers realizations on RMS gains was not addressed.

\section{Conclusions}

We presented a method to compute the slow-switching RMS gain of a switched linear system. The algorithm proposed uses the fact that a given constant $\gamma$ provides an upper bound on the RMS gain whenever there is a separation between all the stabilizing and all the antistabilizing solutions to the algebraic Riccati equations of the systems being switched. We are now in the process of expressing this condition in the form of Linear Matrix Inequalities. The motivation is to construct efficient algorithms that can be used to design switching controllers that minimize the RMS gain of the closed-loop.

One of the implications of this work is the observation that the RMS gain of a switched linear system depends on the realizations of the systems being switched. This is not the case for linear time-invariant systems, for which the RMS gains essentially provide a measure of how much a sinusoidal signal is amplified (ignoring the effects of initial conditions and therefore of realizations). This is because the probe signals that produce maximum energy amplification for linear timeinvariant systems can be chosen to be sinusoids. However, for switched systems this is not the 
case (as demonstrated by the example in the introduction). In fact, for switched systems the probe signals that define the RMS gain may well use transients to maximize energy amplification. Clearly, this must be taken into account when studying questions related to performance and/or robust stability of switched systems.

\section{Appendix}

Proof of (16). To check that $J(\gamma, \sigma)<\infty$ implies that the solution to (13)-(15) exists on every interval $[0, T], T>0$, assume by contradiction that for some $T>0$ the maximum interval of existence of solution is $\left(t_{0}, T\right]$ with $t_{0}>0$ and that $\lim _{t \downarrow t_{0}} x_{0}^{\prime} P(t) x_{0}=+\infty$ for some $x_{0} \in \mathbb{R}^{n}$. Since in this case $V\left(x_{0}, t_{0} ; T, \gamma, \sigma\right)=+\infty$, it is possible to find inputs $u$ defined on $\left[t_{0}, T\right]$ that make $\int_{t_{0}}^{T}\|y(\tau)\|^{2}-\gamma^{2}\|u(\tau)\|^{2} d \tau$ arbitrarily large. Moreover, since the system is controllable for each frozen value of $\sigma$, it is always possible to take the state from $x(0)=0$ to $x\left(t_{0}\right)=x_{0}$ by selecting an appropriate input. This means that we can find inputs $u$ defined on $[0, T]$ that make

$$
\int_{0}^{T}\|y(\tau)\|^{2}-\gamma^{2}\|u(\tau)\|^{2} d \tau
$$

arbitrarily large. Extending these inputs to $+\infty$ by making them zero on $(T,+\infty)$, we conclude that there are inputs that make (35) arbitrarily large for arbitrarily large $T$ and therefore that $J(\gamma, \sigma)=\infty$. Conversely, if the solution to (13)-(15) exists on every interval $[0, T], T>0$ we conclude that for every $T>0$,

$$
\sup _{u \in \mathcal{L}_{2}, x(0)=0} \int_{0}^{T}\|y(\tau)\|^{2}-\gamma^{2}\|u(\tau)\|^{2} d \tau=V(0,0 ; T, \gamma, \sigma)=0
$$

and therefore $J(\gamma, \sigma)=0<\infty$.

Proof of Theorem 1. Because of (18) we conclude from [17, Lemma 4.7] that $\|D\|<\gamma$ and the Hamiltonian matrix $H$ has no eigenvalues on the imaginary axis. Moreover, because $R$ is positive semidefinite and $(A, R)$ is controllable - which is a consequence of the controllability of $(\bar{A}, \bar{B})$ we also have that $P_{1}^{-}$is nonsingular (cf. [17, Theorem 13.6]). From [17, Theorem 13.5], we then conclude that the matrix $P^{-}$defined by (19) is indeed a real, symmetric solution to the algebraic Riccati equation (17) and that $A+R P^{-}$is Hurwitz. Similarly, considering the symmetric algebraic Riccati equation

$$
(-A)^{\prime} P+P(-A)+P(-R) P+(-Q)=0,
$$

(whose Hamiltonian is $-H$ and therefore has the same spectrum as $H$ ) and using the fact that $-R$ is negative semidefinite and $(-A,-R)$ is controllable, we also conclude that $P_{1}^{+}$is nonsingular and therefore the matrix $P^{+}$defined by (19) is a real, symmetric solution to both (36) and (17) and $-A-R P^{+}$is Hurwitz.

Take now a solution $P$ to the algebraic Riccati equation (either $P^{+}$or $P^{-}$). Since $P$ satisfies (17), we have that

$$
\left(\bar{A}+\bar{B} \bar{X}^{-1} \bar{D}^{\prime} \bar{C}\right)^{\prime} P+P\left(\bar{A}+\bar{B} \bar{X}^{-1} \bar{D}^{\prime} \bar{C}\right)+P \bar{B} \bar{X}^{-1} \bar{B}^{\prime} P+\bar{C}^{\prime}\left(I+\bar{D} \bar{X}^{-1} \bar{D}^{\prime}\right) \bar{C}=0,
$$


or equivalently

$$
\bar{A}^{\prime} P+P \bar{A}=-\bar{C}^{\prime} \bar{C}-\left(P \bar{B}+\bar{C}^{\prime} \bar{D}\right) \bar{X}^{-1}\left(\bar{B}^{\prime} P+\bar{D}^{\prime} \bar{C}\right) \leq 0 .
$$

Since $\bar{A}$ is Hurwitz and the pair $(\bar{C}, \bar{A})$ is observable, from standard results on the solution to algebraic Lyapunov equations, we have that

$$
P=\int_{0}^{\infty} e^{\bar{A}^{\prime} t}\left(\bar{C}^{\prime} \bar{C}+\left(P \bar{B}+\bar{C}^{\prime} \bar{D}\right) \bar{X}^{-1}\left(\bar{B}^{\prime} P+\bar{D}^{\prime} \bar{C}\right)\right) e^{\bar{A} t} d t \geq \int_{0}^{\infty} e^{\bar{A}^{\prime} t} \bar{C}^{\prime} \bar{C} e^{\bar{A} t} d t>0 .
$$

Therefore both $P^{+}$and $P^{-}$are positive definite. It remains to show that $P_{+}-P_{-}>0$. We first show that this matrix is nonsingular. To this effect take a vector $x \in \mathbb{R}^{n}$ such that $\left(P_{+}-P_{-}\right) x=0$. From (19), we then conclude that $P_{2}^{-}\left(P_{1}^{-}\right)^{-1} x=P_{2}^{+}\left(P_{1}^{+}\right)^{-1} x$ and therefore

$$
z:=\left[\begin{array}{c}
P_{1}^{-} \\
P_{2}^{-}
\end{array}\right]\left(P_{1}^{-}\right)^{-1} x=\left[\begin{array}{c}
x \\
P_{2}^{-}\left(P_{1}^{-}\right)^{-1} x
\end{array}\right]=\left[\begin{array}{c}
x \\
P_{2}^{+}\left(P_{1}^{+}\right)^{-1} x
\end{array}\right]=\left[\begin{array}{c}
P_{1}^{+} \\
P_{2}^{+}
\end{array}\right]\left(P_{1}^{+}\right)^{-1} x .
$$

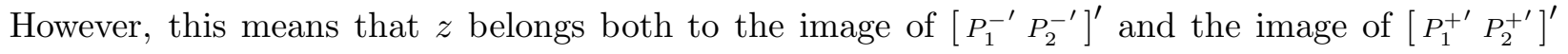
and therefore both to the stable and antistable invariant subspaces of $H$. Since these spaces are independent we must have $z=0$ and therefore $x=0$. This shows that $P_{+}-P_{-}$is nonsingular. On the other hand, since

$$
\begin{aligned}
0=S\left(P^{+}\right)-S\left(P^{-}\right) & =A^{\prime}\left(P^{+}-P^{-}\right)+\left(P^{+}-P^{-}\right) A+P^{+} R P^{+}-P^{-} R P^{-} \\
& =\left(A+R P^{+}\right)^{\prime}\left(P^{+}-P^{-}\right)+\left(P^{+}-P^{-}\right)\left(A+R P^{+}\right)-\left(P^{+}-P^{-}\right) R\left(P^{+}-P^{-}\right),
\end{aligned}
$$

we conclude that

$$
\left(P^{+}-P^{-}\right)^{-1}\left(A+R P^{+}\right)^{\prime}+\left(A+R P^{+}\right)\left(P^{+}-P^{-}\right)^{-1}-R=0 .
$$

Since $-A-R P^{+}$is Hurwitz and $R \geq 0$, we also conclude from standard properties of Lyapunov equations that $\left(P^{+}-P^{-}\right)^{-1} \geq 0$. Since we already established that this matrix is nonsingular, we must actually have $\left(P^{+}-P^{-}\right)^{-1}>0$ and equivalently $P^{+}-P^{-}>0$.

\section{Acknowledgments}

The author would like to thank Dr. G. Zhai for finding an error in previous related work and for subsequent discussions that lead to the results presented here, and to Dr. D. Liberzon, Dr. J. Willems, Dr. Luca Benvenuti, and the anonymous reviewers for several comments that found their way into the final version of the paper. The reference [20] was graciously provided by Dr. E. Jonckheere.

\section{References}

[1] J. P. Hespanha, Encyclopedia of Life Support Systems, ch. Stabilization Through Hybrid Control. UNESCO, 2002. To appear.

[2] A. P. Molchanov and Y. S. Pyatnitskiy, "Criteria of asymptotic stability of differential and difference inclusions encountered in control theory," Syst. \& Contr. Lett., vol. 13, pp. 59-64, 1989. 
[3] K. S. Narendra and J. Balakrishnan, "A common Lyapunov function for stable LTI systems with commuting A-matrices," IEEE Trans. on Automat. Contr., vol. 39, pp. 2469-2471, Dec. 1994.

[4] L. Gurvits, "Stability of linear inclusions-part 2," tech. rep., NECI, Dec. 1996.

[5] T. M. Yoshihiro Mori and Y. Kuroe, "A solution to the common Lyapunov function problem for continuous-time systems," in Proc. of the 36th Conf. on Decision and Contr., vol. 3, pp. 35303531, Dec. 1997.

[6] W. P. Dayawansa and C. F. Martin, "A converse Lyapunov theorem for a class of dynamical systems which undergo switching," IEEE Trans. on Automat. Contr., vol. 44, pp. 751-760, Apr. 1999.

[7] D. Liberzon, J. P. Hespanha, and A. S. Morse, "Stability of switched linear systems: a Liealgebraic condition," Syst. \& Contr. Lett., vol. 37, pp. 117-122, June 1999.

[8] R. N. Shorten and K. S. Narendra, "Necessary and sufficient conditions for the existence of a common quadratic Lyapunov function for two stable second order linear time-invariant systems," in Proc. of the 1999 Amer. Contr. Conf., pp. 1410-1414, June 1999.

[9] R. N. Shorten and K. S. Narendra, "Necessary and sufficient conditions for the existence of a common quadratic Lyapunov function for $m$ stable second order linear time-invariant systems," in Proc. of the 2000 Amer. Contr. Conf., pp. 359-363, June 2000.

[10] A. A. Agrachev and D. Liberzon, "Lie-algebraic stability criteria for switched systems," SIAM J. Contr. Optimization, vol. 40, no. 1, pp. 253-269, 2001.

[11] A. S. Morse, "Supervisory control of families of linear set-point controllers-part 1: exact matching," IEEE Trans. on Automat. Contr., vol. 41, pp. 1413-1431, Oct. 1996.

[12] J. P. Hespanha and A. S. Morse, "Stability of switched systems with average dwell-time," in Proc. of the 38th Conf. on Decision and Contr., pp. 2655-2660, Dec. 1999.

[13] D. Liberzon and A. S. Morse, "Basic problems in stability and design of switched systems," IEEE Contr. Syst. Mag., vol. 19, pp. 59-70, Oct. 1999.

[14] M. Žefran, F. Bullo, and M. Stein, "A notion of passivity for hybrid systems." To appear in the 40th IEEE Conf. on Dec. and Control., Dec. 2001.

[15] J. P. Hespanha, Logic-Based Switching Algorithms in Control. PhD thesis, Yale University, New Haven, CT, 1998.

[16] G. Zhai, B. Hu, K. Yasuda, and A. N. Michel, "Disturbance attenuation properties of timecontrolled switched systems." Submitted to publication., 2001.

[17] K. Zhou, J. C. Doyle, and K. Glover, Robust and Optimal Control. New Jersey: Prentice Hall, 1996.

[18] J. P. Hespanha, "Root-mean-square gains of switched linear systems," tech. rep., University of California, Santa Barbara, Sept. 2002. 
[19] J. C. Willems, "Least squares stationary optimal control and the algebraic Riccati equation," IEEE Trans. on Automat. Contr., vol. AC-16, pp. 621-634, Dec. 1971.

[20] J. M. Rodriguez-Canabal, The Geometry of the Riccati Equation. PhD thesis, University of Southern California, Los Angeles, CA, June 1972.

[21] J. V. Vegte, Feedback Control Systems. New Jersey: Prentice Hall, 3rd ed., 1994.

[22] J. P. Hespanha and A. S. Morse, "Switching between stabilizing controllers," Automatica, vol. 38, Dec. 2002. To appear. 